\title{
GENLOT OPTIMIZATION TECHNIQUES FOR SEISMIC DATA COMPRESSION
}

Laurent C. Duval, Van Bui-Tran

Institut Français du Pétrole

1 et 4 av. de Bois-Préau

92500 Rueil-Malmaison, France

laurent.duval@ifp.fr, van.bui-tran@ifp.fr
Truong Q. Nguyen

Boston University, ECE Dpt.

8 St. Mary's street

Boston MA 02215, USA

nguyent@engc.bu.edu

Trac D. Tran

The Johns Hopkins Univ., ECE Dpt.

3400 N. Charles Street

Baltimore, MD 21218-2686, USA

ttran@ece.jhu.edu

\begin{abstract}
GenLOT coding has been shown an effective technique for seismic data compression, especially when compared to block-based algorithms (such as JPEG), or to wavelets. The transforms remove statistical redundancy and permit efficient compression, when used with advanced encoding techniques, such as the Embedded Zerotree Coding framework. In this work we derive a model for seismic data based on auto-regressive processes. This model is used to design GenLOT filter banks optimized for seismic data, using objective optimization criteria.
\end{abstract}

\section{INTRODUCTION}

Seismic data compression is becoming crucial in geophysical applications, both for storage and transmission purposes. Wavelet coding methods have long been shown effective for compressing seismic data. They have generated interesting developments, including software and hardware implementation for a real-time field test trial in the North Sea in 1995 [SED $\left.{ }^{+} 95\right]$. More recently, methods involving local cosine bases [Mey99], non-unitary filter banks [RRA99] or GenLOTs (generalized lapped orthogonal transforms) have also been developed.

Recent works in image processing have shown than GenLOT with proper design outperform wavelet compression for natural images [TN99]. Some of the authors have demonstrated [DN99] that predesigned GenLOTs outperform state-of-the-art biorthogonal wavelet coders for seismic data. GenLOTs provide a better frequency partioning scheme than wavelets. They act more locally on non stationary seismic data, and tend to better decorrelate the data. Instead of using generic transforms, such as wavelets, it is desirable to adapt the transforms to the data properties.

Røsten et al. have already demonstrated coding gain filter bank optimization for well structured seismic data, such as common offset gathers or stack sections.

In this work, we focus on the problem of compressing raw seismic shots. We propose seismic data modeling based on auto-regressive processes, and subsequent GenLOT optimization using several objective criteria. We conclude that data based optimization is desirable in order to obtain the best from the progressive GenLOT seismic coder described in [DON99]: the resulting transforms yield higher SNRs for the same compression ratio.

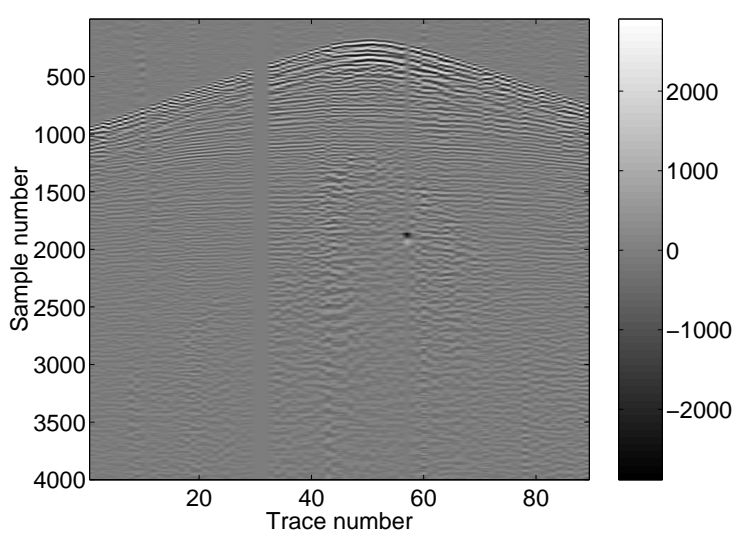

Figure 1: Land raw shot.

\section{MOTIVATIONS OF DATA BASED OPTIMIZATION}

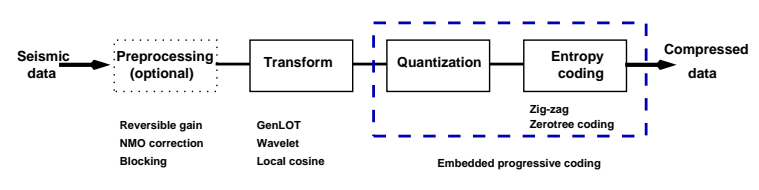

Figure 2: Implementation of a transform based coder.

In most of the transform based natural image coders, the transform stage applies on rows and columns separately. Implementation easiness aside, one reason for this is that natural images are classically modeled as 2-D sets of separable 1-D signals, as exposed in Jayant and Noll's book [JN84]. Evolved coders nevertheless act in a $2-\mathrm{D}$ fashion at least in the entropy coding stage, cf. 
Fig 2, in order to capture more efficiently the 2-D structure of images. Standard JPEG or Said and Pearlman's SPIHT [SP96] make use of 1-D coding (for instance a block DCT or a biorthogonal wavelet) but the JPEG zig-zag order or the Embedded Zerotree Coding introduced by J.-M. Shapiro [Sha93] and extended by Said and Pearlman [SP96] lead to better performance than pure 1-D raster scan coding of the transformed coefficients.

Recent works in image processing have shown than GenLOT with proper design outperform wavelet compression for natural images [?]. Some of the authors have already shown [DN99] that predesigned GenLOTs outperform state-of-the-art biorthogonal wavelet coders for seismic data. GenLOTs provide a better frequency partioning scheme than wavelets, and act more locally on the non stationary seismic data.

We focus here on objective criteria based GenLOT optimization techniques for seismic data compression. Røsten et al. have shown that it is possible to use a separable model for organized 2-D data sets, like common offset gathers or stack sections [Yil87]. In our case, we use raw seismic shots from a land survey. The idea is to find good filter banks and good filter bank optimization techniques for seismic data, without field preprocessing or data sorting, with application to acquisition, in order:

- to avoid or drastically reduce extensive testings of vast amounts of available filter banks by selecting a priori good filters banks;

- to tailor filter banks with degrees of freedom to the statistical properties of the data.

One of the most commonly used objective criterion for filter bank performance is the coding gain. It can be seen as a measure of energy compaction improvement, when using a transform or a subband compression scheme, over a basic pulse code modulation (PCM), as detailed in the Jayant and Noll's book [JN84]. The following chapters demonstrate the use of coding gain along with stopband attenuation and DC leakage optimization for seismic data compression.

The filter banks obtained through optimization in this work are used within the seismic coder proposed by the authors in [DON99].

\section{SIGNAL MODELING}

Let $x$ be a realization of an 1-D real-valued autoregressive process of order $n(\operatorname{AR}(n))$. We assume $x$ has unit variance $\sigma_{x}=1$ and prediction coefficients $b_{1}, b_{2}, b_{3}, \ldots$ Let $r_{x}$ be the normalized auto-covariance function (Acf) of $x$. The right hand side of the Acf also follows a noiseless $\operatorname{AR}(n)$ model, with the same prediction coefficients $b_{1}, b_{2}, b_{3}, \ldots$ For $i \geq n$, we have

$$
r_{x}(i)=b_{1} r_{x}(i-1)+b_{2} r_{x}(i-2)+\ldots+b_{n} r_{x}(i-n) .(1)
$$

The Acf is symmetric (as for any real signal), and the linear equations extend to every lag $i$, as show in Jayant and Noll [JN84] (p. 60-68). In the simple case of an
$\operatorname{AR}(1)$ process, we call $\rho_{1}$ the first normalized coefficient, corresponding to $r_{x}(1)$. The Acf is then given by

$$
r_{x}(i)=\rho_{1}^{|i|}
$$

We will denote by $\operatorname{SNAR}(n)$ a Symmetric Noiseless Autoregressive process with order $n$. In image compression applications, the Acf of these 1-D signals is classically modeled as a SNAR(1), with intersample correlation $\rho_{1}=0.95$ [Mal92, SN96]. Autoregressive models are poorly suited to seismic data in general, but SNAR processes nevertheless fit the seismic data Acf well, as we will see in chapter 4 .

\section{SEISMIC SIGNAL MODELING}

If we try to model seismic signals with an autoregressive process, classical linear progressive coding (LPC) often leads to non stable regression coefficients. One reason could be that seismic signals are often considered as non stationarity. Some consider they still possess some locally stationarity, but this assumption might even be not valid. Nevertheless, if we consider the autocovariance function only, Røsten et al. [RMRP99] have already shown that SNAR(1) or (2) give good results in filter bank optimization.

In the scope of this work, we use SNAR models up order 4 , at which the validity of the model becomes doubtful. We call $\rho_{0}, \ldots, \rho_{3}$ the first four autocovariance coefficients $r_{x}(0), \ldots, r_{x}(3)$. The correlation coefficients are then given by:

$$
\begin{aligned}
\rho_{0} & =1 \\
\rho_{1} & =f\left(b_{1}, b_{2}, b_{3}, b_{4}\right) \\
\rho_{2} & =\frac{b_{2}+\left(b_{1}+b_{3}\right) \rho_{1}}{1-b_{4}} \\
\rho_{3} & =b_{3}+\left(b_{2}+b_{4}\right) \rho_{1}+b_{1} \rho_{2} \\
r_{x}(i) & =b_{1} r_{x}(i-1)+\ldots+b_{4} r_{x}(i-4) .
\end{aligned}
$$

The $b_{i}$ s are obtained directly by calculations on $r_{x}$, and not on the signal $x$ to avoid coefficient instability. The terms $\rho_{0}, \ldots, \rho_{3}$ were found using Eq. 7 and the fact that $r_{x}(-i)=r_{x}(i)$. If we set $b_{1}=b_{2}=b_{3}=0$, and then $b_{2}=b_{3}=0$ we find again the two SNAR models given in Jayant and Noll [JN84]:

$$
\begin{aligned}
r_{x}(0) & =1 \\
r_{x}(1) & =\rho_{1} \\
r_{x}(|i|) & =\rho_{1}^{|i|}
\end{aligned}
$$

and

$$
\begin{aligned}
r_{x}(0) & =1 \\
r_{x}(1) & =\frac{b_{1}}{1-b_{2}} \\
r_{x}(i) & =b_{1} r_{x}(i-1)+b_{2} r_{x}(i-2) .
\end{aligned}
$$

respectively.

There are several ways to obtain the coefficients $b_{i}$. The most convincing method estimates coefficients from 
each horizontal or vertical line of the raw shot, and averages them to obtain an synthetic model of the horizontal and vertical signals, the later displayed in Fig. 3. Modeling results are given in Table 1.

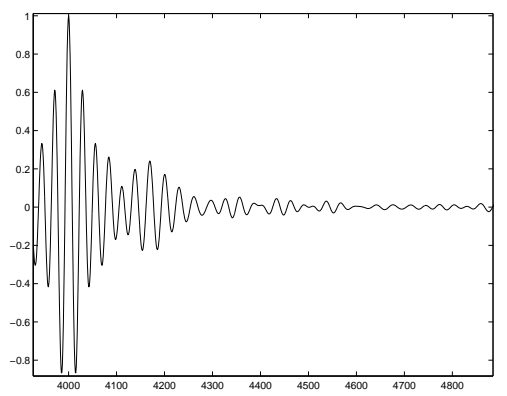

Figure 3: Averaged autocorrelation sample.

\begin{tabular}{lc|cccc} 
Direction & Order & $b_{1}$ & $b_{2}$ & $b_{3}$ & \\
\hline Vertical & 1 & 0.96 & & & \\
& 2 & 1.35 & -0.40 & & \\
& 3 & 1.23 & -0.019 & -0.28 & \\
\hline Horizontal & 4 & 1.17 & -0.023 & -0.015 & -0.21 \\
& 2 & 0.021 & & & \\
& 3 & 0.020 & 0.025 & & \\
& 3 & 0.020 & -0.025 & 0.005 &
\end{tabular}

Table 1: Averaged prediction coefficients at orders 1 to 4

We can see that horizontal correlation is almost inexistent. We thus do not need to use complicated overlapping GenLOT, and a simple DCT is generaly sufficient. The performance of the SNAR models is shown in Fig. 4. They appear to be quite inaccurate, even at small

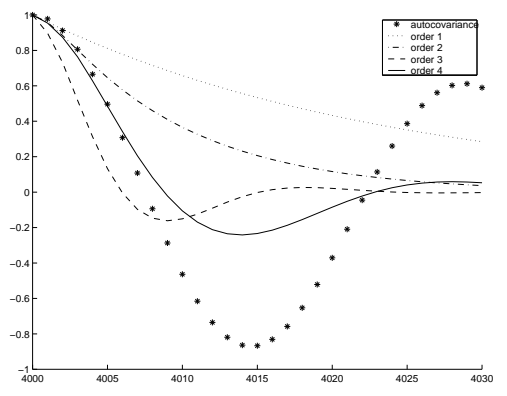

Figure 4: Autocorrelation SNAR models.

lags.

\section{FILTER BANK DESIGN}

Several criteria are used for transform optimization: for instance, coding gain (CG) optimization usually correlates with higher SNRs (objective measure). Other objective measures include stopband attenuation or $D C$ leakage (DC). Though not essential, they often improve the visual quality of the reconstructed data. Since visual quality is not essential to seismic data, we need to caracterize the effect of these measure on seismic signal quality.

\subsection{Coding gain}

Let $x$ be a signal, $\sigma_{x}$ its variance, $\sigma_{x_{i}}$ the variance of the $i$ th subband, and $\left\|f_{i}\right\|$ the $L_{2}$-norm of the $i$ th filter. Under appropriate assumptions, such as optimal bit rate allocation (cf. [RAH95] for a comprehensive survey), the coding gain can be formulated as

$$
C_{C}=10 \log _{10} \frac{\sigma_{x}^{2}}{\prod_{i=0}^{M-1} \sigma_{x_{i}}^{2}\left\|f_{i}\right\|^{2}}
$$

\subsection{Stopband attenuation}

Here, the stopband criterion is chosen to be the contribution of all the filters' energy on $\Omega_{i}$, which defines the outside of $F_{i}$ passband:

$$
C_{S}=\sum_{i=0}^{M-1} \int_{\Omega_{i}}\left|F_{i}\left(e^{j \omega}\right)\right|^{2} d \omega .
$$

\subsection{DC leakage}

The DC leakage measures the part of the DC energy that overlaps out of lowpass subband. It can be defined as:

$$
C_{D}=\sum_{i=0}^{M-1} \sum_{j=0}^{L-1} f_{i}(j) .
$$

These three measures can be varied through appropriate weighting of the following overall cost function $C$ :

$$
C=k_{C} C_{C}+k_{S} C_{S}+k_{d} C_{D} .
$$

We refer to T. Tran's article [?] for more detailed issues on filter bank optimization, and comparison to wavelet coders for natural images.

\section{OPTIMIZATION RESULTS}

In this chapter, we have used a 40-tap, 8-channel structure. The following basic optimization steps have been performed:

1. C: coding gain,

2. D: DC leakage,

3. S: stopband attenuation.

An acronym like CDS4 means the filter bank has been obtained by the C, D, and S steps in this particular order, with implicit SNAR order model 4 for Toeplitz matrix calculation.

Results are given in signal to noise (SNR) vs. compression ratio can be read in tables $2-3$. 


\begin{tabular}{r|ccccc} 
Ratio & C0 & C1 & C2 & C3 & C4 \\
\hline 10 & 69.41 & 71.01 & 70.07 & 70.08 & 70.98 \\
20 & 55.14 & 56.86 & 55.34 & 55.31 & 56.60 \\
30 & 49.62 & 50.11 & 49.93 & 50.05 & 50.33
\end{tabular}

Table 2: Coding gain optimization in the vertical direction vs. DCT.

Table 2 shows that one data based model always performs better than the basic 0.95 intersample correlation. But increasing the order does not always improve quality, and order 1 and 4 perform almost the same. Depending on the compression ratios, order 1 adds between 0.5 and $1.5 \mathrm{~dB}$ approximately.

We have chosen order 1 for further filter bank optimization.

\begin{tabular}{r|ccc} 
Ratio & C1 & CS1 & SCD2 \\
\hline 10 & 71.01 & 71.06 & 70.54 \\
20 & 55.34 & 56.93 & 56.98 \\
30 & 50.11 & 50.22 & 51.38
\end{tabular}

Table 3: Further optimization in the vertical direction.

We can see in Table 3 that filter bank design is not an easy task, since further optimization does not means gain at all compression ratios. Subband attenuation followed by coding gain and DC leakage at order 2 can be down by $0.5 \mathrm{~dB}$ at $10: 1$, but add up to $1.20 \mathrm{~dB}$ at $30: 1$, compared to order 1 optimization.

\section{CONCLUSIONS}

Data based GenLOT optimization is desirable for seismic data, since the simple SNAR models are sufficiently reliable at low prediction orders. We have observed than a "good filter bank" for one particuliar raw shot remain relatively good for other successive raw shots along the same acquisition line. The filter design burden is thus shared by several data sets, and is still useful if we are able to improve the quality by 1 to $3 \mathrm{~dB}$ at a fixed compression ratio.

\section{ACKNOWLEDGMENTS}

The authors would like to thank Steffen Trautmann, who provided us with Matlab ${ }^{\mathrm{TM}}$ optimization routines. Other results on GenLOT optimization can be found at the Waveweb site,

http://saigon.ece.wisc.edu/ waveweb/.

\section{REFERENCES}

[DN99] L. C. Duval and T. Q. Nguyen. Seismic data compression: a comparative study between GenLOT and wavelet compression. In M. Unser, A. Aldroubi, and A. F. Laine, editors, Wavelet Applications in Signal and Image Processing VII. Proc. SPIE 3813, Jul. 1999.

[DON99] L. C. Duval, J. Oksman, and T. Q. Nguyen. A new class of filter banks for seismic data compression. In Proc. 69th Annual SEG Int. Meeting, pages 1907-1910, Oct. 1999.

[JN84] N. S. Jayant and P. Noll. Digital Coding of Waveforms. Prentice-Hall, Inc., Englewood Cliffs, NJ, 1st edition, 1984.

[Mal92] H. S. Malvar. Signal Processing with Lapped Transforms. Artech House, 1992.

[Mey99] F. G. Meyer. Fast compression of seismic data with local trigonometric bases. In M. Unser, A. Aldroubi, and A. F. Laine, editors, Wavelet Applications in Signal and Image Processing VII. Proc. SPIE 3813, Jul. 1999.

[RAH95] T. A. Ramstad, S. O. Aase, and J. H. Husøy. Subbands compression of images: principles and examples. Elsevier, 1995.

[RMRP99] T. Røsten, V. A. Marthinussen, T. A. Ramstad, and A. Perkis. Filter bank optimization for high-dimensional compression of pre-stack seismic data. In Proc. Int. Conf. on Acoustics, Speech, and Signal, March 1999.

[RRA99] T. Røsten, T. A. Ramstad, and L. Amundsen. Part I: Subband coding of common offset gathers. Submitted to Geophysics, 1999.

$\left[\mathrm{SED}^{+}\right.$95] J. P. Stigant, R. A. Ergas, P. L. Donoho, A. S. Minchella, and P. Y. Galibert. Field trial of seismic compression for real time transmission. In Proc. 65th Annual SEG Int. Meeting, pages 960-962, Oct. 1995.

[Sha93] J. M. Shapiro. Embedded image coding using zerotrees of wavelet coefficients. IEEE Trans. on Signal Processing, 41:3445-3462, Dec. 1993.

[SN96] G. Strang and T. Nguyen. Wavelets and Filter Banks. Wellesley-Cambridge Press, Wellesley, MA, 1996.

[SP96] A. Said and W. A. Pearlman. A new fast/efficient image codec based on Set Partitioning in Hierarchical Trees. IEEE Trans. on CAS for Video Technology, 6:243-250, Jun. 1996.

[TN99] T. D. Tran and T. Q. Nguyen. A progressive transmission image coder using linear phase uniform filter banks as block transforms. IEEE Trans. on Image Processing, 8:1493-1507, Nov. 1999.

[Yil87] O. Yilmaz. Seismic data processing. Society of Exploration Geophysicists, 1987. 